\title{
TRATAMENTO DA MÁ OCLUSÃO DE CLASSE II ATRAVÉS DO APARELHO DE PROTRUSÃO MANDIBULAR (APM): UMA REVISÃO DA LITERATURA
}

\author{
Márcio Alexandre de SANTO' \\ bmachris@gmail.com \\ Dênis Clay Lopes dos SANTOS² \\ denis.clay@cruzeirodosul.edu.br \\ Everton FLAIBAN ${ }^{3}$ \\ evertonfaiban@hotmail.com \\ Daniel NEGRETE ${ }^{4}$ \\ daninegrete75@gmail.com
}

Raquel Lopes dos SANTOS 5

raquellopesqls@hotmail.com

\section{RESUMO}

A má oclusão de Classe II pode ser definida como uma relação deficiente entre os arcos dentários, com a cúspide mésio-vestibular do primeiro molar superior ocluindo o espaço entre a cúspide vestibular do primeiro molar inferior e a face distal da cúspide vestibular do segundo pré-molar inferior. A arcada inferior se encontra em relação distal com a arcada superior. Assim, este estudo de revisão tem a finalidade de descrever a má oclusão Classe II com vista no tratamento através do APM (Aparelho de Protrusão Mandibular), com base em estudos epidemiológicos. O delineamento metodológico aplicado para a realização deste estudo foi uma revisão da literatura. Para a busca dos artigos científicos foram utilizadas a base de dados SciELO e revistas nacionais dos últimos anos. Os descritores utilizados foram: Más oclusões de Classe II, APM (Aparelho de Protrusão Mandibular), ortodontia e tipos de tratamento. O Aparelho de Protrusão Mandibular (APM) apresenta uma grande versatilidade clínica, devido à facilidade de fabricação, confecção e ser de baixo custo. Ao longo dos anos vem sofrendo pequenas modificações, mas com objetivo de meIhoramento com vista nas vantagens do seu uso, proporcionando conforto ao paciente.

\section{DESCRITORES: MÁ OCLUSÃO DE ANGLE CLASSE II; TRATAMENTO; APARELHOS ORTODÔNTICOS.}

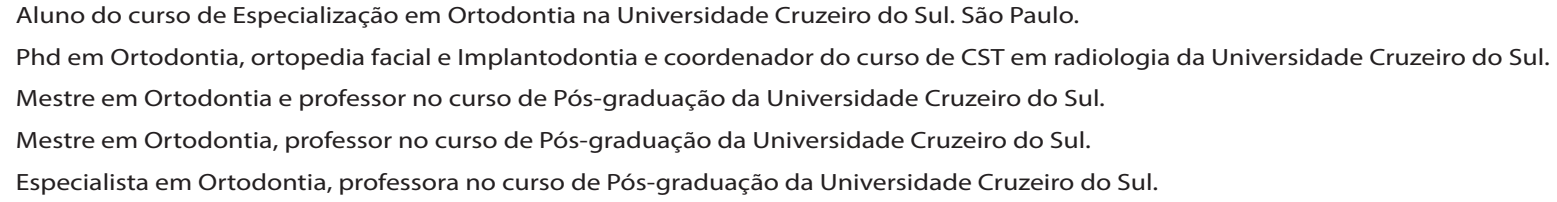


SANTO MA, SANTOS DCL, FLAIBAN E, NEGRETE D, SANTOS RL. Tratamento da má oclusão de Classe II através do aparelho de protrusão mandibular (APM): uma revisão da literatura. Rev. Odontol. Univ. Cid. São Paulo 2018 jul/set 30(3) 304-13

ISSN 1983-5183

\title{
TREATMENT OF CLASS I/ MALOCCLUSION THROUGH THE MANDIBULAR PROTRUSION APPARATUS (APM): A LITERATURE REVIEW
}

\begin{abstract}
Angle's classification is conceptualized as one arcade position in relation to the other in the anterior posterior sense. Class II malocclusion may be defined as a poor relationship between dental arches, with the mesiobuccal cusp of the upper first molar occluding the space between the buccal cusp of the first lower molar and the distal face of the buccal cusp of the second pre-molar ratio. The lower arch is in the distal relation with the upper arch. Thus, this review study has the purpose of describing Class II malocclusion with a view to treatment through the APM (Mandibular Protrusion Apparatus) based on epidemiological studies. The methodological design applied for this study was a review of the literature. For the search of the scientific articles the database was used: SciELO and national journals of the last years. The descriptors used were: Class II malocclusions, APM (Mandibular Protrusion Apparatus), orthodontics and types of treatment. The Mandibular Protrusion Apparatus (APM) presents a great clinical versatility, due to the easy ease in manufacturing and confection and to be of low cost. Over the years it has undergone minor modifications, but with the purpose of improvement with a view to the advantages of its use, providing comfort to the patient.
\end{abstract}

DESCRIPTORS: MALOCCLUSION, ANGLE CLASS II; TREATMENT; ORTHODONTIC APPLIANCES.

\section{INTRODUÇÃO}

A classificação de Angle se conceitua como posição de uma arcada em relação à outra no sentido anteroposterior. A classificação da má oclusão se tornou tradicionalmente um instrumento de grande relevância para um bom diagnóstico e planejamento do tratamento ortodôntico. Desse modo, uma classificação ideal deve sintetizar os dados do diagnóstico e interferir no plano de tratamento'. Para Angle ${ }^{2}$ (1899) a classificação se divide em Classe I, II ou III. Porém, essa classificação não leva em consideração o aspecto facial ou a estética dentária, bem como a severidade da má oclusão.

A má oclusão de Classe II pode ser definida como uma relação deficiente entre os arcos dentários, com a cúspide mésio-vestibular do primeiro molar superior ocluindo o espaço entre a cúspide vestibular do primeiro molar inferior e a face distal da cúspide vestibular do segundo pré-molar inferior. A arcada inferior se encontra em relação distal com a arcada superior ${ }^{3}$.

A Classe II possui 2 divisões: Classe II, 1a divisão, e Classe II, 2a divisão. Uma Classe II subdivisão pode originar-se pela posição distalizada do molar inferior no lado da Classe II ou pela posição mesializada do molar superior no lado da Classe II, sendo que o outro lado se encontra em oclusão normal. Devido às relações oclusais assimétricas, as más oclusões de Classe II subdivisão geralmente apresentam dificuldades de tratamento. A natureza da assimetria oclusal pode ocorrer devido às assimetrias dentoalveolares ou esqueléticas, ou a uma combinação desses elementos, e acredita-se que esses fatores subjacentes ocasionam a obtenção de uma oclusão simétrica ${ }^{4}$.

De acordo com Brunharo et al..$^{5}$ (2006), a Classe II esquelética é um tipo de alteração que pode transcorrer devido a diferentes disposições dos ossos basais, tais como, mandíbula retruída, maxila protruída e/ou uma combinação das duas características ósseas. Por conseguinte, o relacionamento dentário pode se tornar incorreto e estar associado a problemas funcionais e estéticos. 
SANTO MA, SANTOS DCL, FLAIBAN E, NEGRETE D, SANTOS RL. Tratamento da má oclusão de Classe II através do aparelho de protrusão mandibular (APM): uma revisão da literatura. Rev. Odontol. Univ. Cid. São Paulo 2018 jul/set 30(3) 304-13

ISSN 1983-5183

Vale ressaltar que a correção da má oclusão de Classe II, embora não seja a mais frequente na população, constitui a maior prevalência dos casos de pacientes que procuram tratamentos ortodônticos ${ }^{6}$.

No mercado existem diversos aparelhos para o tratamento compensatório desse tipo de oclusão. 0 APM (Aparelho de Protrusão Mandibular) foi elaborado pelo Dr. Coelho filho ${ }^{7}(1995)$, aos moldes do aparelho Herbst. É um aparelho cujo principal objetivo é o posicionamento distal da maxila e o posicionamento mesial da mandíbula. Portanto, apresenta uma eficácia no tratamento compensatório da Classe II por deficiência mandibular, grande versatilidade clínica, fácil confecção e baixo custo ${ }^{8}$.

Assim, a importância da identificação e do tratamento precoce dos indivíduos com má oclusão é relevante para a saúde pública, visto que implica diretamente no custo de tratamento. Dentro desse contexto, as práticas da ortodontia preventiva e interceptativa, mesmo que de modo restrito, podem melhorar a oclusão durante a pré-adolescência e na adolescência ${ }^{9}, 10$.

Desse modo, o plano de tratamento da má oclusão de Classe II é variável, e é importante levar em consideração também a fase de crescimento em que o indivíduo se encontra. Contudo, o estudo irá descrever as características da má oclusão Classe II, causada pela deficiência mandibular, apresentando o aparelho ortopédico e ortodôntico fixo o APM (Aparelho de Protrusão Mandibular) baseados em estudos epidemiológicos através de revisão de literatura.

\section{MÉTODOS}

O delineamento metodológico aplicado para a realização deste estudo foi uma revisão da literatura. Para a busca dos artigos científicos utilizou-se a base de dados: SciELO e revistas nacionais dos últimos anos. Os descritores utilizados foram: Más oclusões de Classe II, APM (Aparelho de Protrusão Mandibular), ortodontia e tipos de tratamento.

\section{REVISÃO DE LITERATURA}

\section{Aparelho de protrusão mandibular: Classe II de Angle}

Em termos epidemiológicos, o termo maloclusão possui alguns sinônimos, tais como oclusopatia" ${ }^{12}$ e má oclusão $0^{13,14}$. Esse agravo à saúde tem recebido crescente destaque, uma vez que ocupa a terceira maior prevalência dentre as doenças bucais, sendo inferior apenas à cárie e à doença periodontal ${ }^{15}$.

A oclusão dentária é considerada como parte morfológica integrante de um sistema fisiológico maior, denominado sistema estomatognático no qual desempenha importantes funçõe ${ }^{16,17}$. As maloclusões, portanto, "representam desvios de normalidade das arcadas dentárias, do esqueleto facial ou de ambos, com reflexos variados tanto nas diversas funções do aparelho estomatognático quanto na aparência e autoestima dos indivíduos afetados"15. Além disso, tem influência sobre a aparência dos portadores de maloclusões, e se tem observado que elas também proporcionam maior risco de traumatismos dentários ${ }^{18}$, os quais, por sua vez, vêm recebendo maior atenção no contexto da odontologia em saúde coletiva, em virtude do referido declínio de cárie observado nos últimos anos ${ }^{19,20,21}$, o que ao mesmo tempo aumenta o potencial de morbidade, através das perdas de elementos dentais, no que condiz a esses traumatismos.

De acordo com a literatura, são variadas as opções de tratamento para a maloclusão de Classe II. Apesar de os objetivos de cada abordagem serem os mesmos, ou seja, melhorar a aparência facial e dentária, manter ou melhorar a saúde oral, e estabelecer um quadro estável de oclusão funcional, as formas 
SANTO MA, SANTOS DCL, FLAIBAN E, NEGRETE D, SANTOS RL. Tratamento da má oclusão de Classe II através do aparelho de protrusão mandibular (APM): uma revisão da literatura. Rev. Odontol. Univ. Cid. São Paulo 2018 jul/set 30(3) 304-13

ISSN 1983-5183

de tratamento são muito diferentes ${ }^{22}$.

Quando se refere aos pacientes em fase de crescimento, a forma de tratamento para a má oclusão Classe II destaca-se na Ortopedia Facial. Os aparelhos ortopédicos funcionais, como o Bionator, o Fränkel e os ativadores, entre outros, agem obtendo uma oclusão funcional e estética satisfatória, reorganizando e readequando os tecidos bucais, proporcionando um crescimento equilibrado entre as bases ósseas ${ }^{23}$.

No início do século passado, Herbst desenvolveu um aparelho fixo para promover a protrusão mandibular, com a finalidade de eliminar o fator cooperação; no entanto, caiu em esquecimento por longos anos em virtude do elevado desenvolvimento dos aparelhos removíveis na Europa e dos elásticos intermaxilares nos Estados Unidos ${ }^{24}$. Porém, em 1979, foi reintroduzido por Pancherz ${ }^{25}$ e então lançado novamente na ortodontia como um fator efetivo para a protrusão mandibular sem a dependência do paciente, proporcionando uma força contínua por 24 horas; nesse sentindo, o APM pode ser considerado um aparelho ortopédico funcional fixo rígido a favorecer ao paciente a possibilidade de não poder removêlo da cavidade bucal e tem como principal objetivo a correção da desarmonia esquelética sagital de Classe Il por meio do avanço mandibular. Portanto, é considerado um aparelho extremamente versátil, devido à indicação para diversas maloclusões e ser extremamente previsível, por promover as alterações esperadas em curto espaço de tempo ${ }^{25}$ para a correção da Classe II, $1^{\text {a }}$ divisão, com retrognatismo mandibular ${ }^{24}$.

Logo após, deu-se início a um desenvolvimento significativo na literatura relacionada às alterações empregadas deste mecanismo e também com relação às modificações e às variações que foram recomendadas ao aparelho original de protração mandibular descrito por Herbst. Conforme Von Bremen e Pancherz ${ }^{26}$ (2002), os dispositivos fixos para o avanço mandibular, quando aplicados na fase de dentadura permanente, são os métodos mais satisfatórios para a correção da Classe II, 1a divisão.

Diante disso, o APM emergiu considerando a escassez de libertação da disciplina e da cooperação do paciente ao utilizar os dispositivos removíveis, como os aparelhos ortopédicos funcionais e o aparelho extrabucal (AEB), concebendo uma circunstância exaustiva entre o profissional e o paciente, além de resultados desagradáveis diante dos casos em que este não colaborava. Em vista disso, Coelho Filho demandava um aparelho que apresentasse as seguintes características ${ }^{25}$ :

-Força distal sobre a maxila;

-Posicionamento mesial da mandíbula;

-Fixo e de uso contínuo, não dependendo da colaboração do paciente;

-Permitindo a abertura e o fechamento da boca, bem como a fala e a mastigação eficientes. As imagens abaixo são de um caso clínico de um paciente com má oclusão de Classe II Divisão 1 com abordagem de APM. (Figura 1 a Figura 3). 
SANTO MA, SANTOS DCL, FLAIBAN E, NEGRETE D, SANTOS RL. Tratamento da má oclusão de Classe II através do aparelho de protrusão mandibular (APM): uma revisão da literatura. Rev. Odontol. Univ. Cid. São Paulo 2018 jul/set 30(3) 304-13

ISSN 1983-5183

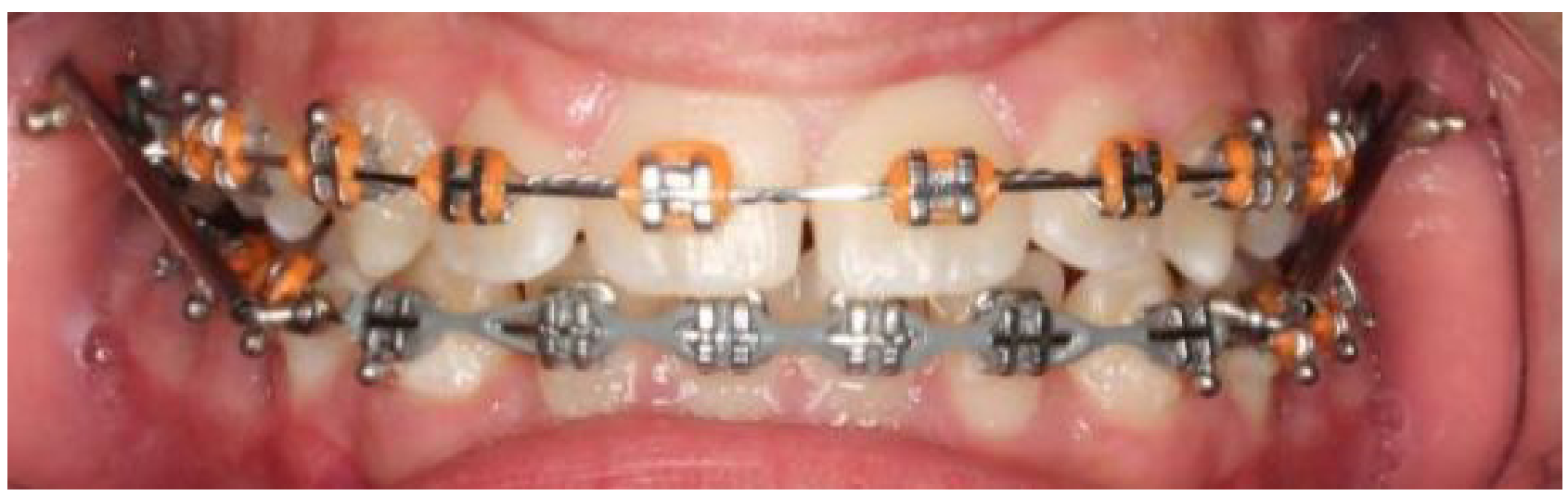

Figura 1- Paciente com uso do APM, foto frontal.

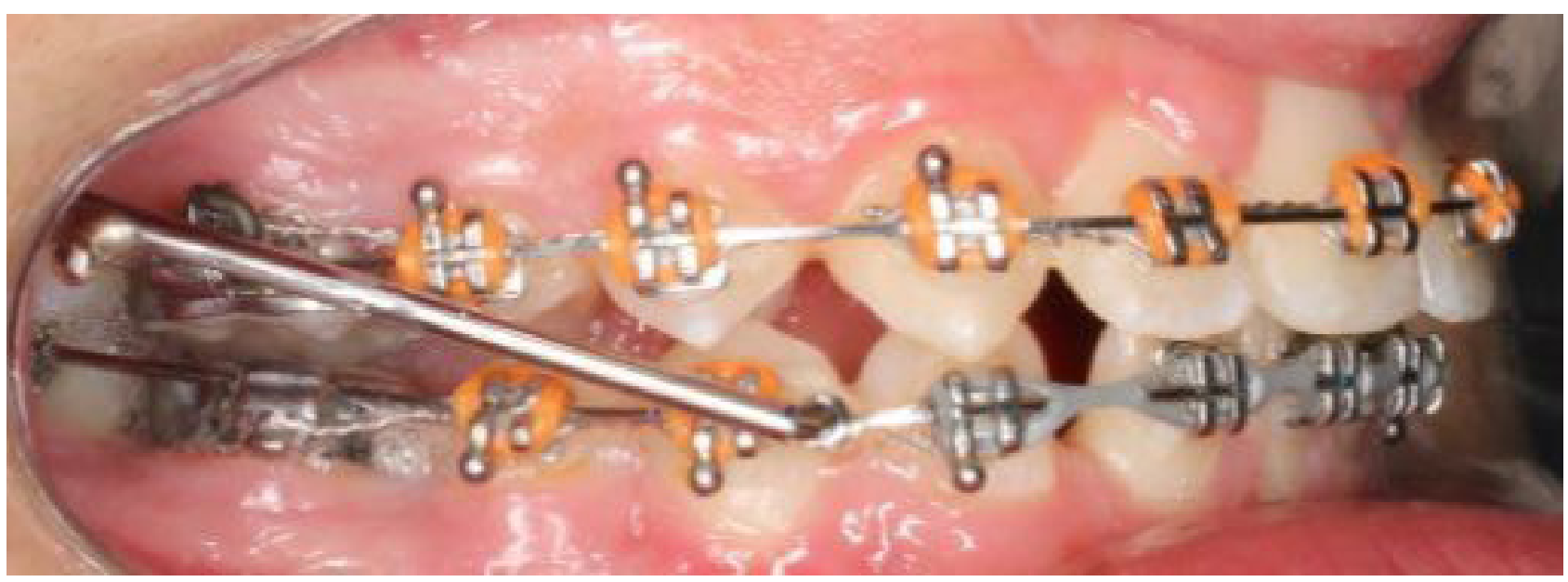

Figura 2 - Paciente com uso do APM, lado direito.

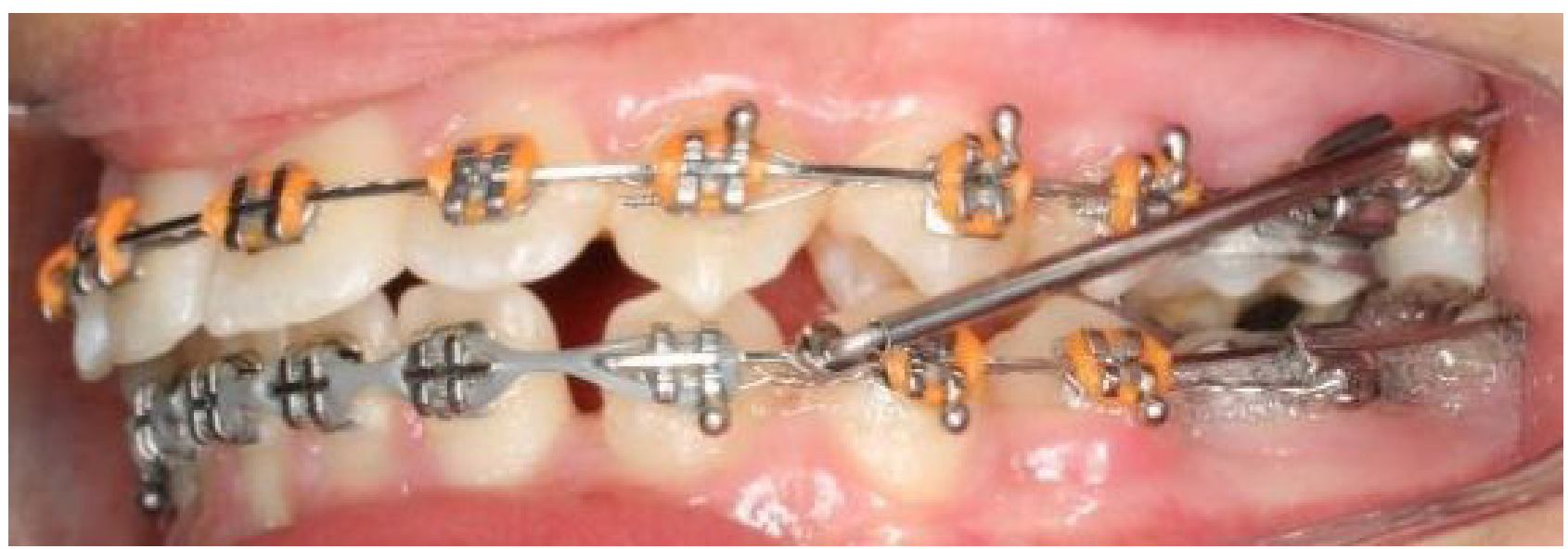

Figura 3 - Paciente com uso do APM, lado esquerdo.

Para $\operatorname{Franco}^{27}(2000)$, o tratamento recomendado da oclusopatia de Classe II, com retrusão mandibular, em pacientes em fase de crescimento é o tratamento ortopédico indicado através do avanço mandibular, de forma a proporcionar o crescimento mandibular e a restringir o crescimento maxilar, objetivando aos pacientes uma vantagem significante no progresso da estética facial com o uso dos aparelhos propulsores mandibulares. É importante ressaltar que a diversidade do uso do APM na prática clínica ortodôntica não se determina somente para o tratamento das Classes II, como também tem demonstrado sua eficiência 
SANTO MA, SANTOS DCL, FLAIBAN E, NEGRETE D, SANTOS RL. Tratamento da má oclusão de Classe II através do aparelho de protrusão mandibular (APM): uma revisão da literatura. Rev. Odontol. Univ. Cid. São Paulo 2018 jul/set 30(3) 304-13

ISSN 1983-5183

nas oclusopatias de Classe I, tais como: preservação de ancoragem dos molares superiores, evitando sua mesialização; distalização de molares superiores; retração em bloco dos dentes superiores; preservação da ancoragem do segmento intercanino inferior, impedindo sua inclinação lingual no processo da mesialização do segmento póstero-inferior nos casos de extração de pré-molares e primeiros molares inferiores; aplicação unilateral ou ativação diferenciada em um dos lados, tanto para correção das relações das Classes Il assimétricas como nos desvios da linha média.

Alves et al. ${ }^{28}$ (2006) demonstraram as vantagens do uso do APM, tais como a utilização do aparelho por 24 hs, não necessita da colaboração do paciente para colocá-lo, um tempo menor de tratamento, não comprometimento estético, o menor custo para o profissional, a facilidade de confecção e fabricação, obediência mínima da colaboração do paciente, assim como a boa aceitação e a versatilidade clínica, sendo indicado em diversos casos.

Freitas e Santos ${ }^{29}$ (2008), em um caso clínico de um paciente com Classe ll completa, divisão l e mordida aberta anterior, apresentaram no plano de tratamento o APM para corrigir essa má oclusão. No resultado do tratamento foi observado que a mordida aberta anterior e a relação molar foram completamente corrigidas.

Conforme estudo de Siqueira et al. ${ }^{30}$ (2011), o avanço mandibular com o APM deve ser realizado até o alcance de uma relação de topo entre os incisivos. Primeiramente, não se deve exceder mais do que $5 \mathrm{~mm}$ de prosseguimento, especialmente por causar desconforto muscular. Caso necessário um novo avanço, é imperioso que seja após dois a três meses da instalação do aparelho e através do segmento de tubo telescópico em comprimento que coincide com a quantidade de acréscimo no avanço mandibular, no lado em que se deseja esse objetivo ou por meio da colocação de mola aberta.

Por outro lado, Moro et al. ${ }^{24}$ (2000) relatam as desvantagens do APM tais como: a rigidez, pode ocorrer interferência na mastigação, a falta de cuidado do paciente pode levar à quebra do aparelho, dificuldade de instalação clínica, não permitir ao paciente total liberdade de movimentos bucais e a falta de colaboração do paciente.

Outras vantagens são pacientes adultos com padrão esquelético Classe II. O estudo de caso foi demonstrado por Bicalho et al..$^{31}$ (2007) em que a paciente se encontrava com deficiência mandibular, relação dentária de Classe II, 2a divisão, 3/4 do lado direito e total do lado esquerdo, mordida cruzada dos dentes 27 e 28 , overjet de $2 \mathrm{~mm}$ e overbite de $3 / 3$ do incisivo inferior; por meio do aparelho de protração mandibular (APM), foi possível realizar modificações dentárias compensatórias complexas numa severa má oclusão de Classe II, 2ª divisão.

Bicalho et al. ${ }^{31}$ (2007) e Furquim et al. ${ }^{32}$ (2013) descrevem que em pacientes adultos o efeito principal do APM se deve somente à compensação dentária com vestibularização dos incisivos inferiores e a mesialização do seguimento anterior. Franco ${ }^{27}(2000)$ descreve que em pacientes em fase de crescimento se faz presente o crescimento mandibular.

Alguns estudos cefalométricos realizados com o objetivo de determinar as alterações dos aparelhos de propulsão mandibular observaram algum impacto do uso dos mesmos na face, principalmente no que diz respeito ao lábio superior, lábio inferior e ângulo nasolabial ${ }^{33,34}$.

Pancherz e Anehus-Pancherz ${ }^{33}$ (1994) avaliaram os efeitos do tratamento com o aparelho Herbst em curto e longo prazo no perfil facial de 69 pacientes Classe II, divisão 1a, tratados por 7 meses e avaliados 
SANTO MA, SANTOS DCL, FLAIBAN E, NEGRETE D, SANTOS RL. Tratamento da má oclusão de Classe II através do aparelho de protrusão mandibular (APM): uma revisão da literatura. Rev. Odontol. Univ. Cid. São Paulo 2018 jul/set 30(3) 304-13

ISSN 1983-5183

após 5 e 10 anos do tratamento. Destes pacientes, 49 exibiram resultados estáveis e 20 casos apresentaram recidiva. Telerradiografias foram tomadas antes do tratamento, após o tratamento, 6 meses após o tratamento e 5 a 10 anos pós-tratamento. Como regra, o tratamento com o aparelho de Herbst resultou na redução da convexidade do perfil facial (perfil mole e duro). O lábio superior retruiu e o inferior se manteve inalterado pela terapia. Durante o período de pós-tratamento de 5 a 10 anos a convexidade do perfil ósseo se reduziu, tanto no grupo com estabilidade como no grupo com recidiva. Quando o nariz foi excluído da avaliação do perfil mole, a convexidade foi reduzida no grupo 16 com estabilidade e permaneceu inalterada no grupo com recidiva. Quando o nariz foi incluído na avaliação, ocorreu um aumento, na média, da convexidade facial nos dois grupos examinados. Os lábios, tanto superiores quanto inferiores ficaram retruídos em ambos os grupos. Os autores concluíram que o aparelho Herbst torna o perfil facial mais reto. O lábio superior ficou retruído enquanto o inferior pareceu não ser afetado pelo tratamento. Porém, houve uma grande variação individual e não previsível.

Diante das variadas vantagens do APM, segundo estudo de Costa e Suguino ${ }^{35}$ (2006), este é um sistema que tem sido utilizado tanto na dentadura mista como na permanente e os resultados demonstrados até então são bastante significativos; dentre os benefícios estão o baixo índice de quebras do aparelho. Além disso, o aparelho permite um amplo movimento de lateralidade, tornando-se extremamente confortável para o paciente.

Os propulsores causam pequenas alterações esqueléticas, mesialização dos molares inferiores e vestibularização dos incisivos inferiores, inclinação distal dos molares superiores e lingualização dos incisivos superiores ${ }^{36,37}$, e favorecem a correção da relação molar. Esse fato deve ser admitido como objetivo do tratamento, já que em indivíduos Padrão II por deficiência mandibular, tratados compensatoriamente, incisivos inferiores com inclinação vestibular aumentada compõem o conceito de normal aceitável ${ }^{1,38} \mathrm{com}$ consequente melhora no perfil, resultado da protrusão do lábio inferior e do pogônio mole, em pacientes com predominância de crescimento horizontal.

\section{CONCLUSÃO}

A má oclusão Classe II é a maior prevalência em casos de pacientes que procuram tratamentos ortodônticos. Diante dos variados tipos de abordagem terapêutica, o estudo destacou o APM por apresentar um novo conceito de tratamento, sendo um dispositivo simples, devido à confecção ser feita pelo próprio profissional, diminuindo o custo do tratamento. Apresenta-se como uma excelente proposta para o tratamento da Classe II como também grande versatilidade clínica, sendo demonstrado como uma alternativa bastante eficaz nos tratamentos compensatórios das más oclusões de Classe II, visto que promove a correção da relação dentária, dependendo de uma menor cooperação do paciente. 
SANTO MA, SANTOS DCL, FLAIBAN E, NEGRETE D, SANTOS RL. Tratamento da má oclusão de Classe II através do aparelho de protrusão mandibular (APM): uma revisão da literatura. Rev. Odontol. Univ. Cid. São Paulo 2018 jul/set 30(3) 304-13

ISSN 1983-5183

\section{REFERÊNCIAS}

1. HENRIQUES JFC, Janson G, Hayasaki SM. Parâmetros para a extração de molares no tratamento ortodôntico: considerações gerais e apresentação de um caso clínico. . Rev Dental Press Ortod Ortop Facial 2002 7(1):57-64.

2. ANGLE EH. Classification of malocclusion. Dental Cosmos 1899 41(2):248-64.

3. ANGLE EH. The latest and best in orthodontic mechanism. Dent Cosmos 1928 70(1):1143-58.

4. AMARAL RMP, Gandini Júnior LG, Gandini MREAS, Mello PB. Tratamento da maloclusão de Classe II, Divisão 1, na dentadura mista com aparelho extrabucal removível. Rev Clin Ortod Dental Press 2011 fev.-mar.;10(1):37-43.

5. BRUNHARO IHP, Mendes AM, Quintão CCA, Fernandes ÁFC, Gravina MA. Classe II esquelética com excesso maxilar: tratamento ortodôntico em duas fases Rev clín ortodon Dental Press 2006 fev.-mar.;5(1):77-82.

6. JANSON G, Barros SEC, Simão TM, Freitas MR. Variáveis relevantes no tratamento da má oclusão de Classe II. Rev Dent Press Ortodon Ortop Facial, Maringá 2009 ago.;14(4):14957.

7. COELHO Filho CM. Mandibular protraction appliances for Class II treatment. J clin orthod : JCO 1995 May;29(5):319-36.

8. CALVEZ X. The universal bite jumper. J clin orthod : JCO 1998 Aug;32(8):493-500.

9. QUEIROZ VS, Nouer PRA, Pereira Neto JS, Magnani MBB, Nouer DF. O Índice de prioridade de tratamento ortodôntico (IPTO) nos diferentes tipos faciais. Ortodontia 2008 out.-dez.;41(4):373-81.

10. GUZZO SC, Finkler M, Reibnitz Júnior C, Reibnitz MT. Ortodontia preventiva e interceptativa na rede de atenção básica do SUS: perspectiva dos cirurgiões-dentistas da prefeitura municipal de Florianópolis. Ciênc saúde coletiva 2014 fev.;19(2):449-60.

11. FRAZÃO P, Narvai PC, Latorre MRDO, Castellanos RA. Prevalência de oclusopatia na dentição decídua e permanente de crianças na cidade de São Paulo, Brasil, 1996. Cad Saúde Pública 2002 out.;18(5):1197-205.

12. PERES KG, Traebert ESA, Marcenes W. Diferenças entre autopercepção e critérios normativos na identificação das oclusopatias. Rev Saúde Pública 2002 abr.;36(2):230-6.

13. TOMITA NE, Bijella VT, Franco LJ. Relação entre hábitos bucais e má oclusão em pré-escolares. Rev Saúde Pública 2000 jun.;34(3):299-303.

14. SILVA Filho OG, Freitas SF, Cavassan AO. Prevalência de oclusão normal e má oclusão em escolares da Cidade de Bauru (São Paulo). Parte I: relação sagital. Rev odontol Univ Sao Paulo 1990 abr.-jun.;4(2):130-7.

15. BRESOLIN D. Índices para maloclusões. In: Pinto, VG. Saúde bucal coletiva. São Paulo: Santos; 2000. p. 197-302. 
SANTO MA, SANTOS DCL, FLAIBAN E, NEGRETE D, SANTOS RL. Tratamento da má oclusão de Classe II através do aparelho de protrusão mandibular (APM): uma revisão da literatura. Rev. Odontol. Univ. Cid. São Paulo 2018 jul/set 30(3) 304-13

ISSN 1983-5183

16. BUENO APF. Sistema estomatognático. In: Bueno, APF. Introdução às bases cibernéticas da ortopedia dentofacial. Rio de Janeiro: Europa; 1991. p. 47-56.

17. SCHURT C. Ortopedia funcional dos maxilares. In: Schurt, C. Ortopedia funcional dos maxilares: da polaridade à unicidade. São Paulo: Quintessence. p. 7-18.

18. FORSBERG CM, Tedestam G. Etiological and predisposing factors related to traumatic injuries to permanent teeth. Swedish dental $j 1993$ 17(5):183-90.

19. FREYSLEBENA GR, Peresb MAA, Marcenes W. Prevalência de cárie e CPO-D médio em escolares de doze a treze anos de idade nos anos de 1971 e 1997, região Sul, Brasil. Rev Saúde Pública 2000 jun.;34(3):304-8.

20. MARCENES W, Bönecker MJS. Aspectos epidemiológicos e sociais das doenças bucais. In: Buischi, YP. Promoção de saúde bucal na clínica odontológica. São Paulo: Artes Médicas; 2000. p. 73-98.

21. NARVAI PC, Castellanos RA, Frazão P. Prevalência de cárie em dentes permanentes de escolares do Município de São Paulo, SP, 1970-1996. Rev Saúde Públic 2000 abr.;34(2):196200.

22. TULLOCH JF, Phillips C, Koch G, Proffit WR. The effect of early intervention on skeletal pattern in Class II malocclusion: a randomized clinical trial. Am J Orthod Dentofac Orthop 1997 Apr;111(4):391-400.

23. FARIA VM, Nogueira PRNR, Shinozaki E, editors. Aparelhos ortopédicos no tratamento das más oclusões de Classe II. VIII Encontro Latino Americano de Pós-Graduação; 2008; Vale do Paraíba: Universidade do Vale do Paraíba.

24. MORO A, Fuziy A, Freitas MR, Henriques JFC, Janson GRP. O aparelho de Herbst e suas variações. Rev Dental Press Ortodon Ortop Facial 2000 mar./abr.;5(2):35-41.

25. PANCHERZ H. Treatment of class II malocclusions by jumping the bite with the Herbst appliance. A cephalometric investigation. Am J Orthod 1979 Oct;76(4):423-42.

26. VON Bremen J, Pancherz H. Efficiency of early and late Class II Division 1 treatment. Am J Orthod Dentofac Orthop 2002 Jan;121(1):31-7.

27. FRANCO AA. Avaliação dos discos das articulações têmporo mandibulares empregando-se imagens por ressonância magnética em pacientes com oclusão normal e maloclusão de classe II, divisão 1, tratados com regulador de função de Frankel [Mestrado]. São Paulo: Faculdade de Odontologia, Universidade Metodista; 2000.

28. ALVES PFR, Oliveira AG, Silveira CA, Oliveira JN, Oliveira Júnior JN, Coelho Filho CM. Estudo comparativo dos efeitos esqueléticos dentários e tegumentares, promovidos pelo tratamento da má oclusão Classe II mandibular com a aparelho de Herbst e com o Aparelho de Protração Mandibular. Rev clín ortodon Dental Press 2006 fev.-mar.;5(1):105.

29. FREITAS BV, Santos PCF. Correção de má-oclusão de Classe II, Divisão 1, com mordida aberta anterior, utilizando-se aparelho de protração mandibular (APM). Rev OrtodontiaSPO 2008 4(1):27. 
ISSN 1983-5183

30. SIQUEIRA DF, Fuziy A, Furquim LZ, Furquim BDA. Versatilidade clínica do aparelho de protração mandibular pro-odonto ortodontia. Pro-Odonto Orto 2011 5(3):9-74.

31. BICALHO JS, Bicalho RF. Utilização do APM no tratamento da má oclusão de Classe II, 2a divisão, em paciente adulto. Rev Clín Ortod Dent Press 2007 fev.-mar.;6(1):99-106.

32. FURQUIM BDA, Henriques JFC, Janson G, Siqueira DF, Furquim LZ. Effects of mandibular protraction appliance associated to fixed appliance in adults. Dental Press $J$ Orthod 2013 out.;18(5):46-52.

33. PANCHERZ H, Anehus-Pancherz M. Facial profile changes during and after Herbst appliance treatment. Eur j orthod 1994 Aug;16(4):275-86.

34. NALBANTGIL D, Arun T, Sayinsu K, Fulya I. Skeletal, dental and soft-tissue changes induced by the Jasper Jumper appliance in late adolescence. The Angle orthodontist 2005 May;75(3):426-36.

35. COSTA LA, Suguino R. Aparelho de protração mandibular: uma nova abordagem na confecção do aparelho. Rev clín ortodon Dental Press 2006 dez. 2005-jan.;4(6):16-28.

36. SIQUEIRA DF, De Almeira RR, Janson G, Brandao AG, Coelho Filho CM. Dentoskeletal and soft-tissue changes with cervical headgear and mandibular protraction appliance therapy in the treatment of Class II malocclusions. Am J Orthod. Dentofac Orthop 2007 Apr;131(4):447.e21-30.

37. HEINIG N, Goz G. Clinical application and effects of the Forsus spring. A study of a new Herbst hybrid. J orofacial orthopedics 2001 Nov;62(6):436-50.

38. CAPELOZZA Filho L. Padrão II. In: Capelozza Filho, L. Diagnóstico em ortodontia. Maringá: Dental Press; 2004. p. 147-234.

RECEBIDO EM 22/05/2018

ACEITO EM 28/09/2018 